\section{Desenvolvimento e validação de equação preditiva da gordura visceral em mulheres jovens}

\section{Development and validation of prediction equations for visceral fat in young women}

\author{
Marina de Moraes Vasconcelos Petribú ${ }^{1}$ \\ Fernando José de Sá Pereira Guimarães \\ Poliana Coelho Cabral ${ }^{3}$ \\ Eduila Maria Couto Santos ${ }^{1}$ \\ Alcides da Silva Diniz ${ }^{3}$ \\ Ilma Kruze Grande de Arruda ${ }^{3}$
}

Resumo - Os métodos atualmente disponíveis para avaliação da obesidade visceral apresentam limitações, tornando-os inviáveis para avaliação em grandes grupos de indivíduos. Esse estudo teve por objetivo desenvolver e validar equação preditiva para estimar a área de tecido adiposo visceral em mulheres jovens. Foram estudadas 64 mulheres entre 19 e 36 anos. As seguintes variáveis foram avaliadas: volume de gordura visceral (VGV) (medido pela ultra-sonografia), Índice de Massa Corporal (IMC), Circunferência da Cintura, Razão Cintura-Estatura (RCE), Razão Cintura-Quadril, Índice de Conicidade, Perfil Lipídico e Glicemia de Jejum (GJ). As mulheres foram divididas em dois grupos: desenvolvimento $(n=48)$ e validação $(n=16)$ da equação. Foram propostas e validadas três equações para estimativa do VGV: Equação 1: VGV=-31,888+(4,044xIMC); Equação 2: VGV=$-51,891+(248,018 x R C E)$; Equação 3: VGV $=-130,941+(198,673$ * RCE $)+(1,185 x G)$, com poder preditivo de $34 \%, 24 \%$ e $45 \%$, respectivamente. Não foi observada diferença estatisticamente significante entre os valores de VGV avaliados pela ultrassonografia e estimados pelas equações preditivas no grupo de validação. As equações propostas podem ser utilizadas para cálculo do VGV de mulheres jovens, quando os exames de imagem não estiverem disponíveis, sendo uma ferramenta valiosa para estudos epidemiológicos e serviços de saúde.

Palavras-chave: Antropometria; Estudos de validação; Gordura intra-abdominal; Obesidade abdominal; Ultrassonografia.

Abstract - The currently available methods for evaluation of visceral obesity have limitations, making them impractical for evaluating large numbers of individuals. This study aimed to develop and validate prediction equations to estimate the area of visceral adipose tissue in young women. The sample included 64 women aged 19 to 36 years. The following variables were evaluated: visceral fat volume (VFV) measured by ultrasound; body mass index (BMI); waist circumference; waist-to-height ratio (WHtR); waist-to-hip ratio (WHR); conicity index; lipid profile; and fasting plasma glucose (FPG). The women were divided into two groups for development $(n=48)$ and validation $(n=16)$ of equations. Three equations were proposed and validated to estimate VFV: Equation 1: VFV $=-31.888+(4.044 \times$ BMI); Equation 2: VFV $=-51.891+\left(248.018^{*}\right.$ WHtR $)$; and Equation 3: VFV $=-130.941+(198.673 \times$ WHtR $)+$ (1.185 $\times$ FPG); with a predictive power of $34 \%, 24 \%$, and $45 \%$ respectively. There was no statistically significant difference between VFV values assessed by ultrasound and estimated by the prediction equations in the validation group. The proposed equations can be used to calculate VFV in young women when imaging tests are not available, providing a valuable tool for epidemiological studies and health services.

Key words: Abdominal; Anthropometry; Intra-abdominal fat; Obesity, Ultrasonography; Validation studies.
1 Universidade Federal de Pernambuco. Centro Acadêmico de Vitória. Núcleo de Nutrição. Vitória de Santo Antão, PE. Brasil.

2 Universidade de Pernambuco. Escola Superior de Educação Física. Recife, PE. Brasil.

3 Universidade Federal de Pernambuco. Centro de Ciências da Saúde. Departamento de Nutrição. Recife, PE. Brasil.

Recebido em 14/09/11 Revisado em 15/11/11 Aprovado em 17/01/12 


\section{INTRODUÇÃO}

A obesidade abdominal é composta de gordura subcutânea e visceral ${ }^{1}$, representando esta última maior valor preditivo para doença cardiovascular ${ }^{2}$. Dentre os métodos utilizados para quantificação do tecido adiposo visceral (TAV) destacam-se Tomografia Computadorizada (TC), Ressonância Nuclear Magnética (RNM) e Ultrassonografia (USG). No entanto, tais métodos apresentam desvantagens, como alto custo, pouca disponibilidade de aparelhos e exposição dos indivíduos à radiação (TC), tornando-os inviáveis para avaliação em grandes grupos de indivíduos ${ }^{3}$.

A facilidade na aplicação de métodos antropométricos, aliada a sua inocuidade, baixo custo e melhor aceitação da população, tornam estes métodos os de maior aplicabilidade na prática clínica e estudos epidemiológicos ${ }^{2}$. No entanto, apesar de diferentes indicadores antropométricos estarem disponíveis para avaliar a gordura abdominal, tais como circunferência da cintura (CC), relação cintura-estatura (RCE), diâmetro abdominal sagital (DAS), índice de conicidade (IC) e relação cintura-quadril (RCQ) $)^{2,4}$, estas medidas não são capazes de diagnosticar separadamente a gordura abdominal subcutânea da visceral ${ }^{5}$. Portanto, pesquisadores tem focado na procura por ferramenta simples, barata e fácil de ser executada para avaliação da adiposidade visceral ${ }^{6,7}$.

Em vista disso, estudos foram realizados com o objetivo de desenvolver e validar equações preditivas para avaliação da obesidade visceral a partir de variáveis antropométricas e exames laboratoriais ${ }^{6,8-10}$. No entanto, existem limitações para aplicabilidade universal dessas equações, já que a distribuição de gordura varia entre sexo, idade e raça ${ }^{11-13}$, tornando evidente que um único método de quantificação não pode ser adotado para mensurar depósitos de TAV da população como um todo. Por isso, este estudo foi desenvolvido com objetivo de desenvolver e validar equações preditivas para estimar o TAV em mulheres jovens.

\section{PROCEDIMENTOS METODOLÓGICOS}

Para cálculo do tamanho amostral, adotou-se valor preditivo de $40 \%$, variabilidade de $12 \%$ e confiabilidade de $95 \%$, resultando numa amostra de 64 mulheres, entre 19 e 36 anos, estudantes da graduação ou pós-graduação da área de saúde da Universidade Federal de Pernambuco, selecionadas por conveniência, em 2009. Da população de estudo, foram excluídas estudantes que haviam realizado cirurgia abdominal recente e gestantes.

A gordura visceral foi estimada pela USG (Logiq 5 US Machine, GE Systems, Milwaukee, WI, USA) realizada com jejum de 8 horas. A gordura visceral

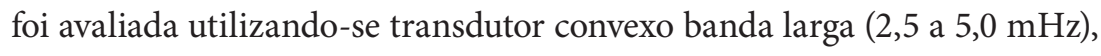
com indivíduos em posição supina após exalação normal. $\mathrm{O}$ volume de gordura visceral (VGV) foi avaliado através da equação proposta por Hirooka et al. ${ }^{14}: \mathrm{VGV}=-9,008+1,191 \mathrm{x}$ [distância entre a superfície interna do músculo reto e da veia esplênica $(\mathrm{mm})]+0,987 x$ [distância entre a superfície interna do mús- 
culo reto e a parede posterior da aorta no umbigo $(\mathrm{mm})]+3,644 \mathrm{x}[$ espessura da camada de gordura da parede renal posterior direita $(\mathrm{mm})]$.

Adotou-se o ponto de corte de $100 \mathrm{~cm}^{2}$ para o diagnóstico da obesidade visceral ${ }^{14}$.

As seguintes medidas antropométricas foram realizadas: massa corporal (balança eletrônica, capacidade $150 \mathrm{Kg}$, divisão $100 \mathrm{~g}$ ), estatura (estadiômetro portátil Ghrum Polar Manufacture, Suíça, precisão 1mm), CC e circunferência do quadril (aferidas com fita métrica não extensível segundo normas da Organização Mundial de Saúde-OMS ${ }^{15}$ ).

A massa corporal e estatura foram mensurados segundo técnicas preconizadas por Lohman et al..$^{16} \mathrm{e}$ utilizadas para cálculo do Índice de Massa Corporal (IMC), classificado de acordo com a OMS ${ }^{15}$. Foram utilizados os índices antropométricos: RCE, RCQ e o IC. A CC e RCQ foram avaliadas utilizando os pontos de corte da $\mathrm{OMS}^{15}$ e para RCE e IC adotaram-se-se os pontos $\geq 0,53^{4} \mathrm{e} \geq 1,18^{4}$, respectivamente.

O perfil lipídico (colesterol total-CT, lipoproteína de baixa densidadeLDL, lipoproteína de alta densidade-HDL, triglicerídeo-TG) e glicemia de jejum (GJ) foram avaliados em sangue venoso, mediante punção cubital, com jejum noturno de 12 horas. Os níveis do perfil lipídico foram determinados por fotometria de absorção com método enzimático. O LDL foi calculado através da fórmula de Friedewald, Levi e Fredrickson (1972) ${ }^{17}$. A GJ foi avaliada através de glucômetro Advantage (Roche Diagnostics). Foram adotados valores de referência citados por Duarte ${ }^{18}$.

$\mathrm{Na}$ análise estatística, realizou-se o teste Shapiro Wilk a fim de verificar a normalidade de distribuição das variáveis contínuas. As mulheres foram divididas em dois grupos: desenvolvimento $(n=48)$ e validação $(n=16)$ da equação. Para identificar quais variáveis seriam incluídas no modelo estatístico, realizou-se análise de correlação de Pearson. Posteriormente, aplicou-se regressão linear múltipla do tipo stepwise e análise residual, adotando-se VGV como variável dependente e as variáveis que apresentaram correlação significativa com o VGV como independentes, iniciando o modelo pela variável preditora que apresentou mais alta correlação com VGV. Para validação das equações, utilizou-se o teste $t$ de Student para amostras pareadas. A fim de testar a concordância entre os valores de VGV medidos através da USG e estimados através da equação 3, realizou-se o teste Bland-Altman ${ }^{19}$.

Adotou-se o nível de significância de 5\% para rejeição da hipótese de nulidade. O pacote estatístico utilizado foi SPSS for Windows (10.0).

O projeto foi aprovado pelo Comitê de Ética do Centro de Ciências da Saúde, da Universidade Federal de Pernambuco (protocolo n067/2008), em 19 de março de 2008. As alunas que concordaram em participar do estudo assinaram o termo de consentimento livre e esclarecido.

\section{RESULTADOS}

A análise descritiva dos dois grupos encontra-se na tabela 1. O grupo 1 
apresenta média de idade superior ao grupo 2, porém ambos são constituídos por mulheres jovens. No que se refere às demais variáveis, não há diferença estatisticamente significativa entre os dois grupos.

Tabela 1. Características gerais da amostra usada para desenvolvimento e validação das equações preditivas da obesidade visceral em mulheres jovens.

\begin{tabular}{|c|c|c|c|c|c|c|c|}
\hline \multirow[t]{2}{*}{ Variável } & \multicolumn{3}{|c|}{ Grupo 1} & \multicolumn{3}{|c|}{ Grupo 2} & \multirow[t]{2}{*}{$\mathrm{P}^{*}$} \\
\hline & $\mathrm{N}$ & Média & DP & $\mathrm{N}$ & Média & DP & \\
\hline Idade (anos) & 48 & 24,02 & 3,78 & 16 & 21,75 & 3,30 & 0,0359 \\
\hline Massa Corporal (Kg) & 47 & 57,23 & 8,30 & 16 & 54,31 & 8,83 & 0,2331 \\
\hline Estatura (m) & 47 & 1,62 & 0,05 & 16 & 1,60 & 0,06 & 0,2296 \\
\hline IMC $\left(\mathrm{Kg} / \mathrm{m}^{2}\right)$ & 47 & 21,95 & 3,36 & 16 & 21,14 & 2,63 & 0,3841 \\
\hline $\mathrm{CC}(\mathrm{cm})$ & 46 & 70,90 & 7,03 & 14 & 68,49 & 4,75 & 0,2353 \\
\hline $\mathrm{CQ}(\mathrm{cm})$ & 44 & 99,16 & 5,63 & 13 & 95,69 & 6,91 & 0,0688 \\
\hline $\mathrm{RCQ}$ & 44 & 0,71 & 0,05 & 13 & 0,71 & 0,02 & 0,9702 \\
\hline RCE & 46 & 0,44 & 0,05 & 14 & 0,43 & 0,03 & 0,4399 \\
\hline IC & 45 & 1,09 & 0,05 & 14 & 1,08 & 0,02 & 0,4406 \\
\hline $\mathrm{GJ}(\mathrm{mg} / \mathrm{dl})$ & 34 & 85,53 & 10,92 & 9 & 83,66 & 3,79 & 0,3688 \\
\hline $\mathrm{CT}(\mathrm{mg} / \mathrm{dl})$ & 35 & 169,68 & 35,23 & 11 & 163,40 & 33,02 & 0,6034 \\
\hline LDL (mg/dl) & 33 & 98,32 & 29,46 & 11 & 88,40 & 18,62 & 0,3016 \\
\hline $\mathrm{HDL}(\mathrm{mg} / \mathrm{dl})$ & 34 & 54,05 & 10,72 & 11 & 55,52 & 19,27 & 0,6322 \\
\hline TG (mg/dl) & 33 & 77,65 & 33,17 & 10 & 97,98 & 47,96 & 0,1342 \\
\hline $\operatorname{VGV}\left(\mathrm{cm}^{2}\right)$ & 48 & 57,20 & 22,47 & 16 & 52,00 & 9,12 & 0,2568 \\
\hline
\end{tabular}

IMC-Índice de Massa Corporal; CC-Circunferência da Cintura; CQ-Circunferência do Quadril; RCQ-Razão CinturaQuadril; RCE-Razão Cintura-Estatura; IC-Índice de Conicidade; GJ-glicemia de jejum; (T-colesterol total; LDLlipoproteína de baixa densidade; HDL-lipoproteína de alta densidade; TG-triglicerídeo; VGV-Volume de Gordura Visceral avaliado através da Ultra-Sonografia; Grupo 1-amostra utilizada para desenvolvimento da equação; Grupo 2-amostra utilizada para validação da equação; DP-desvio padrão; * Teste t de Student para amostras não pareadas.

A tabela 2 descreve os coeficientes de correlação de Pearson das variáveis independentes com relação ao VGV avaliado através da USG no grupo 1. O IMC foi a variável que apresentou maior correlação positiva com o VGV, seguido pela RCE, massa corporal e CC. Estatura, IC e perfil lipídico não apresentaram correlação significativa com o VGV.

Utilizando-se como variável dependente o VGV avaliado através da USG e como variáveis independentes a idade e as variáveis antropométricas que apresentaram correlação significativa com o VGV, obteve-se uma equação antropométrica para estimativa da obesidade visceral (equação 1). Retirando-se o IMC das variáveis independentes incluídas no modelo, obteve-se a equação 2 e incluindo-se a GJ entre as variáveis independentes (excluindo o IMC e a massa corporal), obteve-se a equação 3 (Tabela 3).

O VGV avaliado através da USG e estimado através das três equações preditivas foram medidos no grupo 2 . Não se observou diferença estatisticamente significativa $(\mathrm{p} \geq 0,583)$ entre os valores de VGV avaliados e estimados (Tabela 4). 
Tabela 2. Correlação entre o Volume de Gordura Visceral e idade, variáveis antropométricas, perfil lipídico e glicemia de jejum das estudantes utilizadas no estudo para desenvolver equação preditiva da obesidade visceral.

\begin{tabular}{|c|c|c|}
\hline Variáveis & $\operatorname{VGV}\left(\mathrm{cm}^{2}\right)\left(\mathrm{r}^{*}\right)$ & $\mathrm{P}^{*}$ \\
\hline Idade (anos) & 0,288 & 0,047 \\
\hline Massa Corporal (Kg) & 0,485 & 0,001 \\
\hline Estatura (m) & $-0,257$ & 0,081 \\
\hline $\mathrm{CC}(\mathrm{cm})$ & 0,447 & 0,002 \\
\hline $\mathrm{CQ}(\mathrm{cm})$ & 0,409 & 0,006 \\
\hline IMC $\left(\mathrm{Kg} / \mathrm{m}^{2}\right)$ & 0,580 & 0,000 \\
\hline RCQ & 0,298 & 0,050 \\
\hline RCE & 0,505 & 0,000 \\
\hline IC & 0,105 & 0,493 \\
\hline $\mathrm{GJ}(\mathrm{mg} / \mathrm{dl})$ & 0,377 & 0,028 \\
\hline $\mathrm{CT}(\mathrm{mg} / \mathrm{dl})$ & 0,083 & 0,635 \\
\hline $\mathrm{LDL}(\mathrm{mg} / \mathrm{dl})$ & 0,225 & 0,208 \\
\hline $\mathrm{HDL}(\mathrm{mg} / \mathrm{dl})$ & $-0,122$ & 0,493 \\
\hline TG (mg/dl) & $-0,022$ & 0,904 \\
\hline
\end{tabular}

CC-Circunferência da Cintura; CQ-Circunferência do Quadril; IMC-Índice de Massa Corporal; RCQ-Razão CinturaQuadril; RCE-Razão Cintura-Estatura; IC-Índice de Conicidade; GJ-glicemia de jejum; (T-colesterol total; LDLlipoproteína de baixa densidade; HDL-lipoproteína de alta densidade; TG-triglicerídeo; VGV-Volume de Gordura Visceral avaliado através da Ultra-Sonografia; * Teste de Correlação de Pearson.

Tabela 3. Equações elaboradas para estimar a obesidade visceral a partir de um grupo de mulheres jovens.

\begin{tabular}{|c|c|c|c|c|c|c|c|}
\hline & $\mathrm{N}$ & Modelo* & $\mathrm{p}$ & $r$ & $r^{2} a j$ & $\operatorname{EPE}\left(\mathrm{cm}^{2}\right)$ & $\begin{array}{l}\text { Variáveis independentes } \\
\text { incluídas no modelo }\end{array}$ \\
\hline Equação 1 & 46 & VGV $=-31,888+(4,044 x \mid M C)$ & 0,000 & 0,597 & 0,342 & 18,53 & $\begin{array}{l}\text { Idade, massa corporal, } \\
\text { IMC, CC, RCE }\end{array}$ \\
\hline Equação 2 & 46 & VGV $=-51,891+(248,018 \times R C E)$ & 0,000 & 0,505 & 0,239 & 19,92 & $\begin{array}{l}\text { Idade, massa corporal, } \\
\text { CC, RCE }\end{array}$ \\
\hline Equação 3 & 34 & $\begin{array}{l}\text { VGV }=-130,941+(198,673 \times R C E)+ \\
(1,185 \times J)\end{array}$ & 0,000 & 0,694 & 0,446 & 15,19 & Idade, CC, RCE, GJ \\
\hline
\end{tabular}

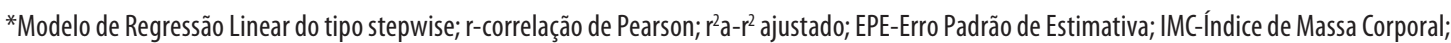
CC-circunferência da cintura; RCE-Razão Cintura Estatura; GJ-Glicemia de Jejum.

Tabela 4. Volume de Gordura Visceral avaliado através da ultra-sonografia e estimado através de três equações preditivas da gordura visceral.

\begin{tabular}{llllll}
\hline & $\mathrm{n}$ & VGV predito pela USG & VGV estimado pela equação & $\mathrm{t}^{*}$ & $\mathrm{p}^{*}$ \\
\hline Equação 1 & 16 & $52,00 \pm 9,12$ & $53,61 \pm 10,64$ & $-0,515$ & 0,614 \\
Equação 2 & 14 & $53,70 \pm 8,28$ & $54,95 \pm 6,80$ & $-0,562$ & 0,583 \\
Equação 3 & 8 & $53,36 \pm 7,94$ & $54,28 \pm 9,79$ & $-0,318$ & 0,760 \\
\hline
\end{tabular}

* Teste $t$ de Student pareado; VGV-Volume de Gordura Visceral; USG-Ultra-sonografia; Equação 1-VGV = -31,888+(4,044xIMC); Equação 2-VGV = -51,891+(248,018xRCE); Equação 3-VGV = -130,941+(198,673xRCE)+( 1,185xGJ); IMC-Índice de Massa Corporal; RCE-Razão Cintura Estatura; GJ-Glicemia de jejum.

A Figura 1 apresenta o gráfico de Bland-Altman, mostrando a dispersão dos erros obtidos através da equação 3 ao estimar o VGV, quando comparado com os valores obtidos através da USG. Observa-se uma boa concordância entre os dois métodos, com viés próximo a zero. 


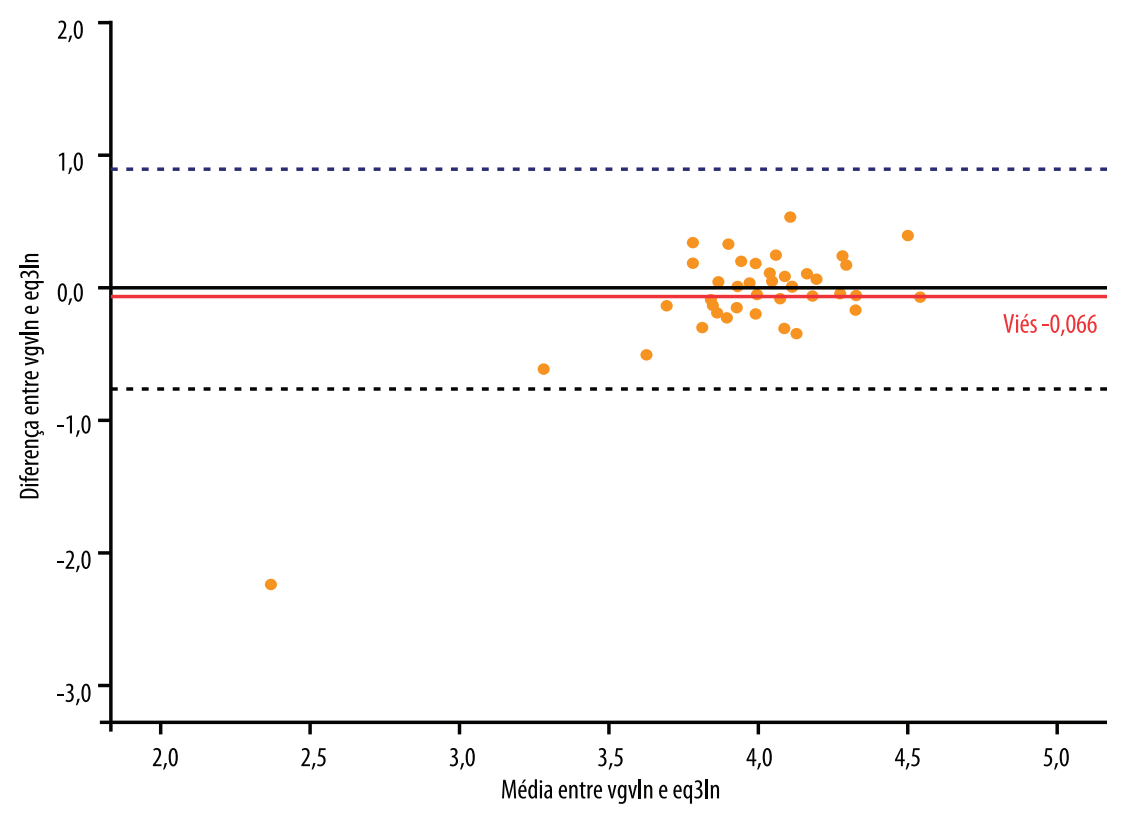

Vgvln = logaritmo natural do volume de gordura visceral avaliado através da Ultra-Sonografia; eq3ln = logaritmo natural do volume de gordura visceral estimado através da equação preditiva

Figura 1. Concordância entre a Ultrassonografia e a equação preditiva da gordura visceral analisada através do Teste de Bland Altman

\section{DISCUSSÃO}

A importância das equações propostas e validadas no presente estudo se dá pelo fato do perfil de deposição de gordura ser específico para cada sexo e etnia, além de sofrer influência da idade 2 . A amostra foi constituída apenas por mulheres jovens, todas brasileiras. A literatura relata que mulheres tendem a apresentar área de TAV menor do que homens, apesar destas apresentarem maior área de gordura subcutânea ${ }^{20}$. Além disso, indivíduos jovens tendem a armazenar a massa gorda subcutaneamente ${ }^{21}$, sendo citado que $\mathrm{o}$ TAV acumula-se com aumento da idade ${ }^{22,23}$. Com relação à raça/etnia, já foi observado que mulheres e homens brancos, caucasianos e hispânicos apresentam maior TAV quando comparados aos negros e afro-americanos ${ }^{11-13}$.

Embora não tenham sido estudadas raça e etnia das mulheres incluídas na atual pesquisa, a maioria da população brasileira é composta por indivíduos miscigenados, pois desde a chegada dos primeiros colonos portugueses assistiu-se à miscigenação em massa com índios e décadas depois, com escravos negros, formando-se uma população tri-híbrida. Portanto, acredita-se que a população brasileira apresenta uma particularidade em relação à raça, possuindo contribuição genética europeia, africana e de povos indígenas, além de uma diversificação étnica e cultural.

Apesar de outros estudos terem proposto equações para estimar o $\mathrm{VGV}^{6,8-10}$, estes foram desenvolvidos em populações de raças diferentes, muitos com o sexo masculino e a maioria para faixa etária superior à da presente pesquisa, não tendo sido encontrado nenhum estudo realizado numa população brasileira jovem do sexo feminino. 
Ao incluir apenas variáveis antropométricas no modelo, a equação 1 foi desenvolvida, no entanto, tal equação apresentou como variável independente o IMC. Apesar de alguns estudos também terem encontrado correlação moderada e significativa entre o IMC e a gordura visceral ${ }^{24,25}$, outros encontraram resultados divergentes ${ }^{1,26}$. Sampaio ${ }^{1}$ mostrou que o IMC foi o indicador antropométrico menos adequado para discriminar o TAV em ambos os sexos. Além disso, Despres et al. ${ }^{26}$ chamam a atenção para o fato de o IMC não ter sido considerado preditor significante e independente da variação de TAV em nenhuma das equações desenvolvidas em seu estudo, após a inclusão de outras variáveis antropométricas.

A utilização do IMC para avaliação do estado nutricional vem sendo questionada ${ }^{2,21}$. Este índice representa mais um indicador de massa corporal do que propriamente de adiposidade, uma vez que não distingue massa magra de massa gorda; além disso, correlaciona-se de forma significante com a estatura e a massa livre de gordura ${ }^{21}$, sendo incapaz de avaliar a distribuição da gordura corporal ${ }^{2}$.

Portanto, apesar da equação com o IMC ter apresentado melhor poder de predizer a gordura visceral e menor erro padrão de estimativa, quando apenas variáveis antropométricas foram incluídas no modelo, a incapacidade do IMC em avaliar a distribuição da gordura corporal ${ }^{2}$ limitou a utilização desta equação. Sendo assim, um segundo modelo foi criado após a não inclusão do IMC dentre as variáveis independentes. Tal modelo apresentou como variável independente a RCE, concordando com o estudo de Nagai et al. ${ }^{9}$ que propuseram uma equação para predizer o VGV de homens usando como variáveis a RCE e o TG, apresentando alta sensibilidade e especificidade. A razão da CC com a estatura pode ser mais fortemente correlacionada aos fatores de risco para doenças coronarianas do que a CC isolada ${ }^{27}$.

A principal limitação de tais equações diz respeito ao baixo poder preditivo ( $34 \%$ e $24 \%$, respectivamente), reforçando a ideia de que outros fatores, além de medidas antropométricas, contribuem para variação no TAV, como descrito por Nagai et al. ${ }^{9}$, que encontraram que um modelo de regressão utilizando dados de uma avaliação médica é mais preciso do que um usando apenas índices antropométricos.

Na tentativa de melhorar o poder preditivo, a GJ foi incluída no modelo. Esta equação apresentou a vantagem de predizer o VGV em quase $45 \%$, com valor mais próximo dos encontrados na literatura (52 a 74\% ${ }^{8,20,26}$ ), além de apresentar menor erro padrão de estimativa. Porém, sua desvantagem está relacionada ao menor tamanho amostral utilizado para desenvolvimento e validação, tendo em vista a ausência de dados laboratoriais em alguns indivíduos da amostra. Chama atenção, ainda, o fato de 55\% do VGV não ter sido explicado pelas variáveis incluídas no modelo. Després et al. ${ }^{26} \mathrm{re}-$ forçam que outros fatores, além da gordura corporal total e distribuição do tecido adiposo subcutâneo, tais como os fatores genéticos, são determinantes significantes do TAV, dificultando a tentativa de estimá-lo. Demerath ${ }^{28}$ ressalta que os efeitos genéticos podem explicar 40 a $60 \%$ na variação do TAV. 
Diferentemente do estudo realizado por Despres et al. ${ }^{26}$, que teve a idade incluída em seu modelo como uma das variáveis independentes, o efeito da contribuição da idade para a predição do VGV não foi evidente no presente estudo. Tal fato pode ser explicado pela pequena amplitude da faixa etária dos sujeitos envolvidos no estudo, portanto, o efeito biológico da mobilização da gordura periférica para a centralização e internalização pode não ter ocorrido ainda.

Este estudo apresenta como limitações o pequeno número de participantes da amostra, resultado da limitação de recursos financeiros, da pequena faixa etária, do fato de apenas mulheres terem sido incluídas no estudo e da não avaliação da raça e etnia das participantes. Além disso, a gordura visceral foi aferida através da USG, e não através da TC (atual padrão ouro) ou RNM, pelo fato de a TC expor os indivíduos à radiação ionizante e a RNM ter um custo extremamente elevado ${ }^{14}$. No entanto, já foi demonstrado que o TAV avaliado através da USG apresenta uma boa correlação com o medido pela TC, já tendo sido validado como instrumento de avaliação da gordura visceral ${ }^{14}$, o que justificou sua adoção como método de referência.

\section{CONCLUSÕES}

Apesar das limitações do presente estudo, acredita-se que as equações propostas e validadas podem ser utilizadas para estimar o VGV de mulheres jovens, quando exames de imagem não estiverem disponíveis, sendo uma ferramenta valiosa para estudos epidemiológicos e serviços de saúde. Dentre as equações, a que apresenta melhor aplicabilidade é a terceira, tendo em vista sua maior capacidade de predizer o VGV e menor erro padrão de estimativa.

Mais estudos devem ser realizados visando à obtenção de outras equações que possam ser utilizadas em diferentes faixas etárias e no sexo masculino, além de equações que incluam outras variáveis de fácil obtenção e que apresentem um poder explicativo da variabilidade do TAV superior a estas.

\section{REFERÊNCIAS BIBLIOGRÁFICAS}

1. Sampaio LR, Simões EJ, Assis AM, Ramos LR. Valididy and reliability of the sagittal abdominal diameter as a predictor of visceral abdominal fat. Arq Bras Endocrinol Metab 2007;51(6):980-6.

2. Vasques ACJ, Priore SE, Rosado LEFPL, Franceschini SCC. Utilização de medidas antropométricas para avaliação do acúmulo de gordura visceral. Rev Nutr 2010;23(1):107-18.

3. Ribeiro-Filho FF, Mariosa LS, Ferreira SRG, Zanella MT. Gordura visceral e síndrome metabólica: mais do que uma simples associação. Arq Bras Endocrinol Metab 2006;50(2):230-8.

4. Haun DR, Pitanga FJG, Lessa I. Razão cintura/ estatura comparado a outros indicadores antropométricos de obesidade como preditor de risco coronariano elevado. Rev Assoc Med Bras 2009;55(6):705-11.

5. Hayashi T, Boyko E, McNeely M, Leonetti DL, Kahn S, Fujimoto W. Minimum waist and visceral fat values for identifying Japanese Americans at risk for the metabolic syndrome. Diabetes Care 2007; 30: 120-7. 
6. Gauralet M, Hernandez-Morante JJ, Tebar FJ, Zamora S, Canteras M. Two-dimentional predictive equation to classify visceral obesity in clinical practice. Obesity 2006;14(7):1181-91.

7. Rankinen T, Kim SY, Perusse L, Despres JP, Bouchard C. The prediction of abdominal visceral fat level from body composition and anthropometry: ROC analysis. Int J Obesity 1999;23:801-9.

8. Goel K, Gupta N, Misra A, Poddar P, Pandey RM, Vikram NK, et al. Predictive equations for body fat and abdominal fat with DXA and MRI as reference in Asian Indians. Obesity 2008;16(2):451-6.

9. Nagai M, Komiya H, Mori Y, Ohta T. Developments in estimating visceral fat area from medical examination data. J Atheroscler Thromb 2008; 15 (4): 193-8.

10. Demura S, Sato S. Prediction of visceral fat area in Japanese adults: proposal of prediction method applicable in a field setting. Eur J Clin Nutr 2007;61(6):727-35.

11. Stanforth PR, Jackson AS, Green JS, Gagnon J, Rankinen T, Despres JP, et al. Generalized abdominal visceral fat prediction models for black and white adults aged 17-65y: the HERITAGE Family Study. Int J Obes Relat Metab Disord 2004; 28(7):925-32.

12. Hill JO, Sidney S, Lewis CE, Tolan K, Scherzinger AL, Stamm ER. Racial differences in amounts of visceral adipose tissue in young adults: the CARDIA study. Am J Clin Nutr 1999;69(3):381-87.

13. Carroll JF, Chiapa AL, Rodriguez M, Phelps DR, Cardarelli KM, Vishwanatha JK, et al. Visceral fat, waist circumference, and BMI: impact of race/ ethnicity. Obesity 2008;16(3):600-7.

14. Hirooka M, Kumagi T, Kurose K, Nakanishi S, Michitaka K, Matsuura B, et al. A technique for the measurement of visceral fat by ultrasonograhy: comparison of measurements by ultrasonography and computed tomography. Internal Med 2005;44(8):794-99.

15. World Health Organization. Obesity: preventing and managing the global epidemic. Report of a WHO consultation on obesity. Geneva; 1998. Available from: https:// apps.who.int/nut/documents/obesity_executive_summary.pdf. [2010 jul 03].

16. Lohman TG. Roche AF, Martorell R. Anthropometric standardization reference manual. Abridged, Champaing, Illinois. Human Kineticis Books, 1991.

17. Friedewald WT, Levi RI, Fredrickson DS. Estimation of the concentration of low density lipoproteins cholesterol in plasma without use of the ultracentrifuge. Clin Chem 1972;18:499-502.

18. Duarte ACG. Interpretação laboratorial na prática nutricional ambulatorial. In: Duarte ACG, editor. Avaliação nutricional: Aspectos clínicos e laboratoriais. São Paulo: Atheneu, 2007. p. 501-530.

19. Hirakata VN, Camey SA. Análise de concordância entre métodos de Bland-Altman. Rev HCPA 2009;29(3):261-8.

20. Brundavani V, Murthy SR, Kurpad. Estimation of deep-abdominal-adipose-tissue (DAAT) accumulation from simple anthropometric measurements in Indian men and women. Eur J Clin Nutr 2006;60:658-66.

21. Cervi A, Franceschini SCC, Priore SE. Análise crítica do uso do Índice de Massa Corporal para idosos. Rev Nutr 2005;18(6):765-75.

22. Schwartz RS, Shuman WP, Bradbury VL, Cain KC, Fellingham GW, Beard JC, et al. Body fat distribution in healthy young and older men. J Gerontol 1990;45:M181-5.

23. Kotani K, Tokunaga K, Fujioka S, Kobatake T, Keno Y, Yoshida S, et al. Sexual dimorphism of age-related changes in whole-body fat distribution in the obese. Int J Obes 1994;18:207-12.

24. Kamel EG, McNeill G, Han TS, Smith FW, Avenell A, Davidson L, et al. Measurement of abdominal fat by magnetic resonance imaging, dual-energy X-ray absorptiometry and anthropometry in non-obese men and women. Int J Obes Relat Metab Disord 1999; 23(7):686-92. 
25. Radominski RB, Vezozzo DP, Cerri GG, Halpern A. O uso da ultra-sonografia na avaliação da distribuição de gordura abdominal. Arq Bras Endocrinol Metabol 2000;44(1):5-12.

26. Després JP, Prud'homme D, Pouliot MC, Tremblay A, Bouchard C. Estimation of deep abdominal adipose-tissue accumulation from simple anthropometric measurements in men. Am J Clin Nutr 1991;54:471-7.

27. Ashwell M, Cole TJ, Dixon AK. Ratio of waist circumference to height is strong predictor of intra-abdominal fat. Br Med J 1996;313:559-60.

28. Demerath EW. Causes and consequences of human variation in visceral adiposity. Am J Clin Nutr 2010;91:1-2.
Endereço para correspondência

Marina de Moraes Vasconcelos Petribú Rua do Alto do Reservatório, s/n, Bela Vista, CEP: 55608-680-Vitória de Santo Antão, PE. Brasil

E-mail: mpetribu@hotmail.com 